\title{
Mechanisms of Autocatalytic Multistage Structure Formation Reactions in Dairy Based Systems in Relation to Processing and Compositional Factors
}

\author{
Ulrich Kulozik $^{1}$, Stefanie Sedlmeier ${ }^{1}$, Sonja Lenze ${ }^{2}$ \\ 1. Chair of Food and Bioprocess Engineering, School of Life Sciences, Technical University of Munich, \\ Freising-Weihenstephan, Germany \\ 2. New affiliation: Hochland AG, Heimenkirch, Germany
}

A multistage reaction is described, which seems to be typical for gel formation in high protein model systems (Fig. 1). It is the objective of this study to identify the influencing factors behind the reaction and to identify the mechanisms related to its stages, i.e. the effects of compositional parameters and operational conditions on the structural and rheological changes characterizing the reaction, commonly referred to as creaming reaction. The underlying reaction conditions are phenomenologically known [1; 2], but still insufficiently scientifically understood so far.

The hypothesis was that both proteins and fat are involved in the structure formation, with protein as the active component and fat in an indirect role. The effect of the proteins' molecular or structural status, protein content and type, fat content and fat globule size were studied in this work. Specifically, the effects of fat level, protein content (Fig. 2) as well as the effect of rework addition, on the course of the structure development during the creaming reaction were investigated. The status of both, protein (micellar, molecular, partially disintegrated) and fat (globule size) were modified by targeted upstream treatment.

By using a rheometer with customized geometry as a micro-reactor and as analytical inline tool we identified critical process times, at which the system changes its state for a certain time before again being converted by the reaction conditions such that the next stage in structure formation is induced. By chromatographic techniques we analyzed structural changes on the molecular level. We found that certain minima in fat and protein level are required for the multistage structure formation to occur. By means of light microscopy we showed that originally larger emulsified fat globules are reduced in size on the course of reaction, despite low shear rates. Obviously, the firmer structure, which is based on developing protein network formation, leads to gradually increasing shear stresses over time and, thus, to the formation of smaller fat globules (Fig. 3). The decisive point in time appears to have been reached at 76 min (stage C). By means of upstream homogenisation of the fat phase and lower incoming fat droplet size, the structure formation can be accelerated. Rework, i.e. product elements already processed once and added to the next product lot, also has a tremendous influence accelerating the course of the structure formation. Electron microscopy indicated that a network of protein strings is formed, apparently sourced by protein, before located at the fat droplet surfaces as emulsifier. We conclude that two parallel or partially sequential reactions are taking place, inducing the formation of a finely dispersed emulsion as well as a protein network in the continuous phase. Compositional and other processing related factors including the addition of rework affect the structure formation process, rework probably acting as a starter in a self assembly reaction.

References:

[1] G Dimitreli and AS Thomareis, Journal of Food Engineering 64 (2004), p. 265-271.

[2] SK Lee et al, Journal of Food Science and Technology 36 (2003), p. 339-345. 


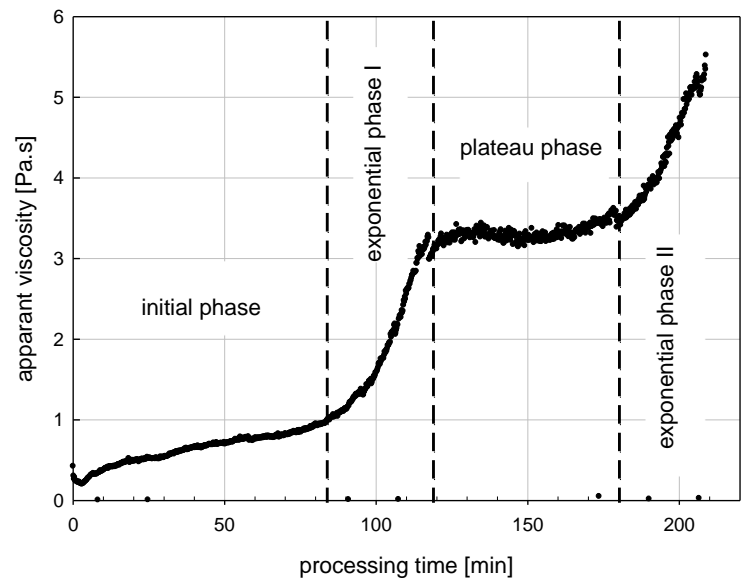

Figure 1. Characteristic course of structure formation

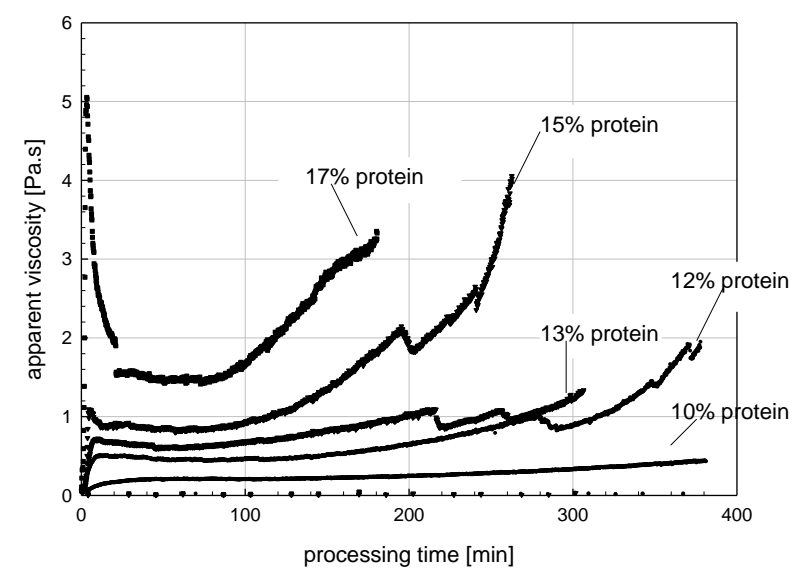

Figure 2. Effect of protein content on the structure formation
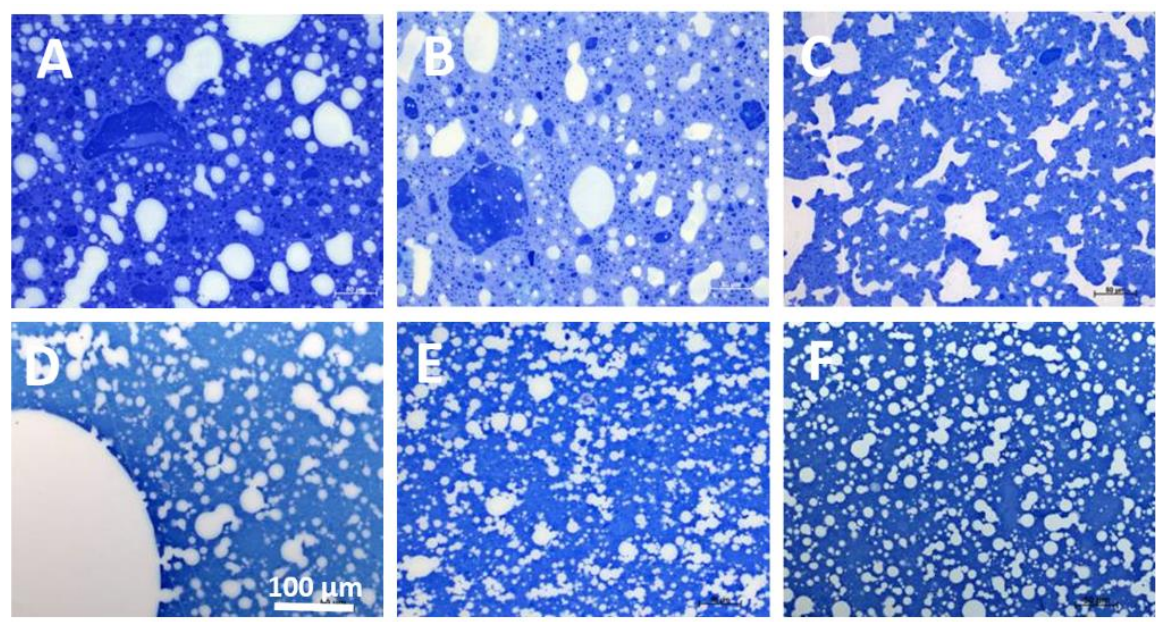

Figure 3. Light microscopical analysis of samples along the reaction course; A: $30 \mathrm{~min}, \mathrm{~B}$ : $60 \mathrm{~min}, \mathrm{C}$ : 76 min, D: 100 min, E: 120 min, F: 150 min 